\title{
In Situ Structure and Assembly of the Bacterial Multidrug Efflux Pump
}

Xiaodong Shi ${ }^{1}$, Muyuan Chen ${ }^{1}$, James Michael Bell ${ }^{2}$, Zhili Yu ${ }^{1}$, Steven J. Ludtke ${ }^{1}$ and Zhao Wang ${ }^{1,3 *}$

1. Verna and Marrs McLean Department of Biochemistry and Molecular Biology, Baylor College of Medicine, Houston, Texas, USA.

2. Graduate Program in Quantitative and Computational Biosciences, Baylor College of Medicine, Houston, Texas, USA

3. Department of Molecular and Cellular Biology, Baylor College of Medicine, Houston, Texas, USA.

* Corresponding author: zhaow@bcm.edu

With the increasing use of antibiotics, multidrug resistance in pathogenic bacteria has grown to become a medical crisis to public health. Multidrug efflux pumps play an important role in conferring drug resistance, as they are capable of expelling a variety of toxic substrates from the cell. Targeting assembly of efflux pumps is a promising strategy to overcoming antibiotic resistance [1]. In Gramnegative bacteria, these pumps form tripartite assemblies that span both the inner and outer membranes making them difficult for traditional structural studies [2].

AcrAB-TolC is one of the tripartite pumps that are constitutively expressed in Escherichia coli (E. coli) [3]. As the main multidrug efflux machinery, AcrAB-TolC is comprised of the outer membrane protein TolC, the periplasmic adaptor protein AcrA, and the inner membrane transporter AcrB from the Resistance-Nodulation-cell Division (RND) superfamily [2]. The AcrAB-TolC efflux pump transports diverse compounds, conferring resistance to a broad spectrum of antibiotics [4]. Structural studies of this pump have been limited to individual components by X-ray crystallography [5-8] or fully assembled pumps by cryo-electron microscopy (cryo-EM) single particle analysis $[9,10]$.

We have now visualized the first in situ structure of AcrAB-TolC efflux pump at $15 \AA$ resolution in bacteria cell using electron cryo-tomography and subtomogram averaging. There is long time ambiguity about assembly process of tripartite pump in bacteria, our study for the first time, observed partially assembled complexes and their contacts with the peptidoglycan layer. In addition, the fully assembled efflux pump was visualized in an "open state" in the presence of AcrB inhibitor and a "closed state" in the presence of antibiotic, suggesting that the efflux of antibiotic is a transient cellular process in contrast to complexes in vitro. Taken together, these results reveal the assembly mechanism of tripartite efflux pumps in living bacteria and provide the structural basis for future therapeutic investigations of drug efflux pumps.

\section{References:}

[1] K Poole, J Antimicrob Chemother 56 (2005), p. 20.

[2] D Du et al., Curr Opin Struct Biol 33 (2015), p. 76.

[3] D Ma et al., Mol Microbiol 16 (1995), p. 45.

[4] H Okusu, D Ma and H Nikaido, J Bacteriol 178 (1996), p. 306.

[5] V Koronakis et al., Nature 405 (2000), p. 914.

[6] J Mikolosko et al., Structure 14 (2006), p. 577.

[7] T Eicher et al., Proc Natl Acad Sci U S A 109 (2012), p. 5687.

[8] MA Seeger et al., Science 313 (2006), p. 1295.

[9] D Du et al., Nature 509 (2014), p. 512. 
[10] Z Wang et al., eLife 6 (2017), p.

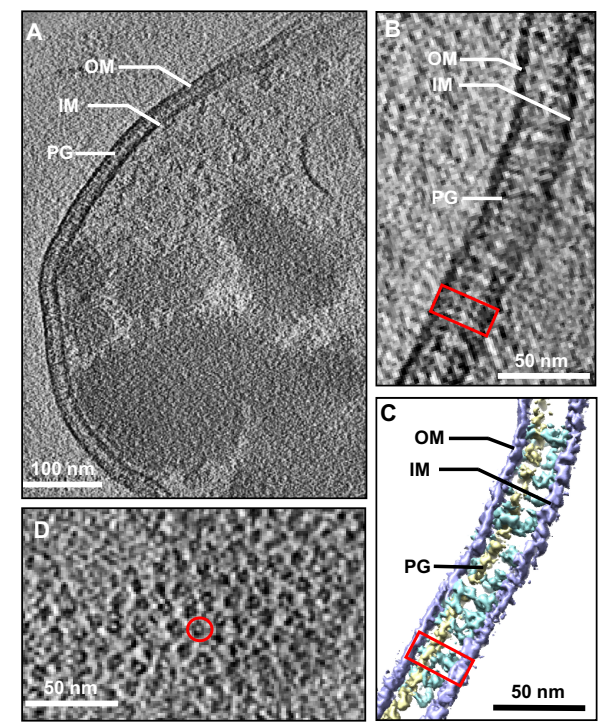

Figure 1. Visualizing the AcrAB-TolC efflux pump in the E. coli cell envelope. (A) A single slice from a tomogram of E. coli. (B) Zoomed in side view of the cell envelope containing the AcrAB-TolC pump. (C) Corresponding three-dimensional annotation of (B) showing the outer membrane (OM; blue), the inner membrane (IM; blue), peptidoglycan (PG; yellow), and the AcrAB-TolC pump (cyan). (D) Top view of the cell envelope containing the AcrAB-TolC pumps which appear as ring-shaped densities.

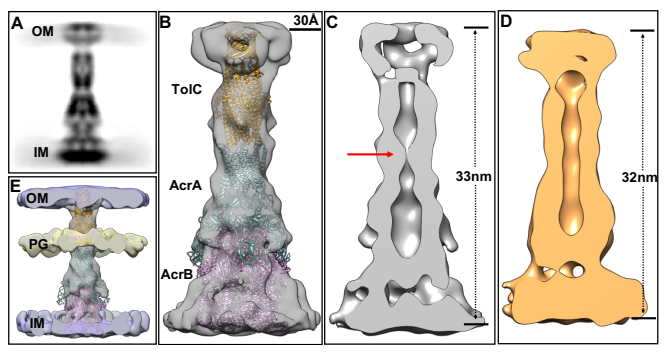

Figure 2. Cryo-ET structures of the fully assembled AcrAB-TolC complex in situ. (A) The side-view projection of the subtomogram average of the pump in presence of antibiotics. (B) Isosurface rendering of (A) fitted with high resolution cryo-EM model. (C) A slice through (B) showing a constriction at the boundary between AcrA and TolC (indicated by the red arrow). (D) A slice through the density map of the pump in presence of AcrB inhibitor. (E) Isosurface rendering of (B) overlaid with density map of the cell envelope. 